Research Article

\title{
Construction of a Novel Doxorubicin Nanomedicine Using Bindarit as a Carrier: A Multiscale Computer Simulation-Assisted Design-Based Study
}

\author{
Anjie Shi $\left(\mathbb{D},{ }^{1}\right.$ Ling Long $\left(\mathbb{D},{ }^{2}\right.$ Zhibin Liu $\left(\mathbb{D},{ }^{1}\right.$ Yao Liu $\left(\mathbb{D},{ }^{1}\right.$ Qingjia Gong $(\mathbb{D})$,

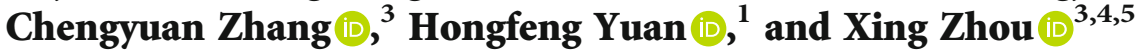 \\ ${ }^{1}$ Department of Ophthalmology, Army Medical Center of PLA, Army Medical University, Chongqing 400042, China \\ ${ }^{2}$ Department of Oncology, Xinqiao Hospital, Army Medical University, Chongqing 400039, China \\ ${ }^{3}$ School of Pharmacy and Bioengineering, Chongqing University of Technology, Chongqing 400054, China \\ ${ }^{4}$ Department of Pharmacy, Chongqing Hospital of Traditional Chinese Medicine, Chongqing 400011, China \\ ${ }^{5}$ Chongqing Key Laboratory of Medicinal Chemistry \& Molecular Pharmacology, Chongqing University of Technology, \\ Chongqing 400054, China
}

Correspondence should be addressed to Hongfeng Yuan; yhf871@sina.cn and Xing Zhou; diszhou@cqut.edu.cn

Received 9 September 2021; Accepted 9 October 2021; Published 27 October 2021

Academic Editor: Ruibing Wang

Copyright (c) 2021 Anjie Shi et al. This is an open access article distributed under the Creative Commons Attribution License, which permits unrestricted use, distribution, and reproduction in any medium, provided the original work is properly cited.

\begin{abstract}
Nanomedicines typically use polymeric materials or liposomes as carriers. This provides targeting advantages but may lead to a series of defects, such as low drug loading, high risk in terms of safety, and high production costs. Herein, we report a computer simulation-assisted designing method for the construction of a novel doxorubicin (DOX) nanomedicine without any polymer carriers. We used a small molecular drug, bindarit (BIN), as a carrier of DOX to provide synergistic antitumor effects. First, the intermolecular forces between DOX and BIN were calculated for evaluating the interaction and potential conformation of the DOX/BIN complex. Then, the potential assembly ability of the DOX/BIN complex was predicated here by using dissipative particle dynamic stimulation. These computational simulation results suggested that BIN could form an amphiphilic complex with DOX through $\pi-\pi$ stacking, hydrogen bonding, and electrostatic interaction and then self-assemble to nanoaggregates at the mesoscopic scale. Under the computational guidance, doxorubicin/bindarit nanoparticles (DOX/BIN NPs) in a spherical morphology were successfully prepared, and these NPs possess the original cytotoxic activity of DOX. Thus, this multiscale computer simulation-assisted design strategy can serve as an effective approach to develop nanomedicines using small molecules as a carrier.
\end{abstract}

\section{Introduction}

Chemotherapy is a widely used strategy for the treatment of tumors. Traditional chemotherapeutic drugs usually have evident disadvantages such as toxicity to normal cells, poor precision, and multidrug resistance [1]. Currently, an increasing number of traditional chemotherapeutic drugs are prepared as nanomedicines, which provides improved antitumor efficiency and reduced side effects, compared to the drugs alone [2]. These nanomedicines are usually prepared with a large amount nonactive excipients as carriers, such as liposomes [3, 4], polymers [5], and inorganic nanoparticles [6]. However, the use of these excipients as basic materials for the formation of nanomedicines may lead to several problems, such as low drug loading, high risk in terms of safety, and high production costs [7]. These limitations negatively impact the prospects of these nanomedicines in clinical use. Therefore, researchers are committed to developing strategies to reduce the proportion of inactive substances in nanomedicines through supramolecular assembly and other approaches, to achieve higher drug loading, increased drug safety, and simpler preparation methods. 
In our previous study [8], we successfully engineered a paclitaxel (PTX) nanoaggregate without any inactive excipients, using the small active molecule, indomethacin, as a carrier [9]. Compared to nanomedicines using polymers or other inactive excipients as carriers, this PTX nanomedicine showed natural advantages in drug loading and safety. Similar to PTX, doxorubicin (DOX) is an effective and extensive chemotherapeutic drug; however, its toxicity poses a significant clinical challenge [10]. Pegylated liposomal doxorubicin has been approved by the Food and Drug Administration for clinical use having a safer profile but at a much higher cost than the common DOX injection $[11,12]$. Although numerous novel DOX nanomedicines have been developed, most of them are facing obstacles for clinical application based on the excipients. Bindarit (BIN) is a classic inhibitor of monocyte chemotactic protein-1 [13], which can reduce the infiltration of tumor-associated macrophages to inhibit tumor progression and metastasis [14-17]. BIN and DOX are polyaromatic compounds with a carboxyl group and an amino group, respectively [18]. They have strong intermolecular forces because of hydrogen bonds and $\pi-\pi$ stacking. Notably, BIN is a potentially appropriative drug carrier for DOX; however, whether BIN possesses the ability to load DOX remained unknown.

In recent years, computer-aided technologies such as molecular docking and molecular dynamics have been widely applied in the screening and design of new drugs, by predicting the intermolecular interactions between drugs and targets, and the conformation of drug-target complex [19-21]. However, the application of these computer-aided technologies in designing nanomedicines is still in its infancy. In our previous studies [8], we have successfully employed these computeraided technologies for analyzing the intermolecular forces driving the assembly of nanomedicines.

Herein, we hypothesize that these computer-aided technologies can also be used in designing nanomedicines. Taking BIN and DOX as model drugs, a computer simulation from the atomic to the mesoscopic scale was designed and successfully utilized to engineer a novel DOX nanomedicine using BIN as a carrier. Under the computer-aided guidance, DOX/BIN NPs with inherited antitumor activity from DOX were successfully prepared.

\section{Materials and Methods}

\subsection{Computer Simulation Methods}

2.1.1. AutoDock. The molecular formulas of DOX and BIN were downloaded from DrugBank (https://www.drugbank .ca/) and optimized through geometry optimization in Materials Studio 2017 software (Accelrys Inc.). In AutoDock 4.2 [22], the optimized molecular conformation of BIN was imported as a ligand, and the optimized molecular conformation of DOX was imported as a macromolecule. The grid box was set to $126 \AA \times 126 \AA \times 126 \AA$, and the distance of each small grid point was $0.0375 \mathrm{~nm}$. Lamarckian GA [23], a genetic algorithm, simulated annealing algorithm, and local search algorithm were used for calculations, and all other parameters remained unchanged, by default.
2.1.2. DPD Simulation. First, DOX, BIN, and water molecules were set to the corresponding beads $(\mathrm{D}, \mathrm{B}$, and $\mathrm{W}$, respectively) in Materials Studio 8.0. The Flory-Huggins interaction parameters between these beads were calculated using the Blends module. According to the formula $\alpha_{\mathrm{ij}}(\rho=$ $3)=25+3.50 \cdot \chi^{2}$, the results are summarized in a table as the parameters of the DPD simulation [24].

After the mesoscopic molecules were constructed, a mesoscopic model of DOX, BIN, and water molecules was built at a DOX/BIN/water ratio of $1: 1: 8$. To optimize the mesoscopic model, we first created a force field using the DPD method with a length scale of $6.46 \AA$ and a mass scale of $54 \mathrm{amu}$ and then replaced the values of the DPD simulation parameters. After the mesoscopic model was optimized, DPD simulations were performed for 50 ns. After the DPD simulations, the molecular distances between the core of DOX/BIN NPs and DOX, BIN, and water was calculated using the radial distribution function.

\subsection{Preparation of $D O X / B I N$ NPS}

2.2.1. Materials. DOX was purchased from Seebio (Shanghai, China). Bindarit (BIN) was obtained from Nanjing Chemlin Chemical Industry Co., Ltd. (Nanjing, China). Dialysis members were purchased from Repligen (Boston, MA, USA).

2.2.2. Preparation of Hydrophobic DOX. Doxorubicin hydrochloride was dispersed in ultrapure water with ten equivalents of triethylamine, the solution was continuously stirred overnight at $4^{\circ} \mathrm{C}$ in the dark, and hydrophobic doxorubicin was obtained after drying.

2.2.3. Preparation. Dialysis was performed to produce the DOX/BIN NPs. Firstly, $5 \mathrm{mg}$ of hydrophobic doxorubicin and $5 \mathrm{mg}$ of BIN were dissolved in $1 \mathrm{~mL}$ of dimethyl sulfoxide (DMSO). Subsequently, the solution was transferred to a dialysis bag, which was dialyzed against deionized water at $25^{\circ} \mathrm{C}$. The outer aqueous solution was exchanged every half an hour for 6 times and then exchanged every 1 hour for the next 5 times. Finally, after 8 hours of dialysis, the samples were collected and dried for further analysis. The drug loading content was calculated using the following equation:

$$
\begin{aligned}
\text { Drug loading content }= & \frac{\text { the weight of drug in nanoparticles }(\mathrm{mg})}{\text { the weight of nanoparticles }(\mathrm{mg})} \\
& \times 100 \% .
\end{aligned}
$$

2.3. Characterization of DOX/BIN NPs. The distribution of particle zeta-potential and the diameter of the NPs were measured using a Malvern Zetasizer Nano ZS instrument (Malvern, UK). Ultraviolet spectra were recorded using a UV-visible spectrophotometer (TU-1810, Beijing, China). Fourier transform infrared spectra (FT-IR) were measured using Fourier transform infrared spectroscopy (VERTEX70, Bruker, Germany). Transmission electron microscopy (TEM) observations were performed using a Tecnai-10 
microscope (Philips, Netherlands). All samples were examined at $20^{\circ} \mathrm{C}-25^{\circ} \mathrm{C}$.

2.4. Cytotoxicity Assays. Y79 human retinoblastoma cell lines were obtained from the American Type Culture Collection (ATCC, USA). Because Y79 cells do not adhere to the bottom of the plate, the 96 -well plate was coated with $50 \mathrm{mg} / \mathrm{mL}$ poly-D-lysine solution (Beyotime, Shanghai, China) to facilitate adherence. Y79 cells at a density of $5 \times 10^{3}$ cells/well were inoculated with $100 \mu \mathrm{L}$ RPMI-1640 medium (containing $10 \%$ fetal bovine serum, $1 \%$ penicillin-streptomycin) and cultured in $5 \% \mathrm{CO}_{2}$ at $37^{\circ} \mathrm{C}$ for 24 hours. After aspirating the supernatant from each well, $100 \mu \mathrm{L}$ of medium containing reagent (DOX/BIN NPs or DOX) was added for $48 \mathrm{~h}$. After removal of the medium, $100 \mu \mathrm{L}$ of $10 \%$ CCK-8/RPMI $1640(v / v)$ per well was added for $2 \mathrm{~h}$. Then, the absorbance at $450 \mathrm{~nm}$ was measured using a microplate reader (Biotek, Synergy H1, Winooski, VT, USA). All experiments were performed in quadruplicate. The relative cell viability was calculated using the following equation:

$$
\text { Cell viability }(\%)=\frac{A(\text { test group })}{A(\text { control group })} \times 100 \% \text {. }
$$

\section{Results and Discussion}

3.1. The Intermolecular Forces between DOX and BIN. It is well known that a strong intermolecular interaction is the key to the formation of stable intermolecular complexes. Thus, the interactions between DOX and BIN were initially calculated using AutoDock 4.2. As shown in Table 1, according to Lamarckian GA, the intermolecular energy was $-8.03 \mathrm{kcal} / \mathrm{mol}$, which was contributed by van der Waals forces (Vdw) and hydrogen bonds (H-bonds) between DOX and BIN. The results of the other three algorithms (genetic algorithm, simulated annealing algorithm, and local search algorithm) were similar. Mediated by this strong intermolecular interaction between DOX and BIN, a stable BIN/DOX complex was formed after BIN docking to DOX. Subsequently, the interactions between DOX and DOX as well as BIN and BIN were calculated. As shown in Tables 2 and 3, according to Lamarckian GA, the intermolecular energy was $-6.94 \mathrm{kcal} / \mathrm{mol}$ and $-5.29 \mathrm{kcal} / \mathrm{mol}$ for DOX-DOX and BIN-BIN, respectively, both interactions less than that between DOX and BIN. As shown in Figure 1, the result suggested that DOX tended to combine with BIN rather than itself.

As shown in Figure 2, the intermolecular interactions between DOX and BIN were mainly composed of $\pi-\pi$ stacking interaction and $\mathrm{H}$-bonds. The $\pi-\pi$ stacking interaction was attributed to the conjugated aromatic groups in DOX and BIN, while $\mathrm{H}$-bonds were contributed by the hydroxyl group in DOX and the carboxyl group in BIN. Additionally, through the hydrophilic and hydrophobic surface rendering of the DOX/BIN complex, we can clearly see that the BIN/DOX complex shows an obvious amphiphilic property. The amino group of DOX and the carboxyl group of BIN constitute the hydrophilic region, while conjugated aromatic groups constitute the main hydrophobic region (Figure 2).
TABLE 1: Molecular docking results between DOX and BIN (unit: $\mathrm{kcal} / \mathrm{mol}$ ).

\begin{tabular}{lcccc}
\hline & $\begin{array}{c}\text { Lamarckian } \\
\text { GA }\end{array}$ & $\begin{array}{c}\text { Genetic } \\
\text { algorithm }\end{array}$ & $\begin{array}{c}\text { Simulated } \\
\text { annealing }\end{array}$ & $\begin{array}{c}\text { Local } \\
\text { search }\end{array}$ \\
\hline Binding energy & -5.94 & -3.97 & -3.05 & -4.22 \\
Intermol energy & -8.03 & -6.06 & -5.14 & -6.31 \\
Vdw & -6.08 & -4.57 & -4.58 & -4.64 \\
Electrostatic energy & -1.95 & -1.49 & -0.56 & -1.67 \\
Ligand efficiency & -0.25 & -0.17 & -0.13 & -0.18 \\
Inhibit constant & 44.47 & 1.22 & 5.84 & 810.18 \\
Total internal & 1.21 & 1.26 & -0.83 & -1.42 \\
Torsional energy & 2.09 & 2.09 & 2.09 & 2.09 \\
Unbound energy & 1.21 & 1.26 & -0.83 & -1.42 \\
H-bond & 1 & 2 & 1 & 1 \\
\hline
\end{tabular}

TABLE 2: Molecular docking results between DOXs (unit: kcal/mol).

\begin{tabular}{lcccc}
\hline & $\begin{array}{c}\text { Lamarckian } \\
\text { GA }\end{array}$ & $\begin{array}{c}\text { Genetic } \\
\text { algorithm }\end{array}$ & $\begin{array}{c}\text { Simulated } \\
\text { annealing }\end{array}$ & $\begin{array}{c}\text { Local } \\
\text { search }\end{array}$ \\
\hline Binding energy & -3.64 & -2.56 & -1.11 & -2.81 \\
Intermol energy & -6.93 & -5.84 & -4.39 & -6.09 \\
Vdw & -6.51 & -5.59 & -4.34 & -5.70 \\
Electrostatic energy & -0.41 & -0.25 & -0.05 & -0.39 \\
Ligand efficiency & -0.09 & -0.07 & -0.03 & -0.07 \\
Inhibit constant & 2.13 & 13.31 & 152.69 & 8.75 \\
Total internal & -6.22 & -5.74 & -3.63 & -4.20 \\
Torsional energy & 3.28 & 3.28 & 3.28 & 3.28 \\
Unbound energy & -6.22 & -5.74 & -3.63 & -4.20 \\
H-bond & 1 & 1 & 1 & 1 \\
\hline
\end{tabular}

TABLE 3: Molecular docking results between BINs (unit: kcal/mol).

\begin{tabular}{lcccc}
\hline & $\begin{array}{c}\text { Lamarckian } \\
\text { GA }\end{array}$ & $\begin{array}{c}\text { Genetic } \\
\text { algorithm }\end{array}$ & $\begin{array}{c}\text { Simulated } \\
\text { annealing }\end{array}$ & $\begin{array}{c}\text { Local } \\
\text { search }\end{array}$ \\
\hline Binding energy & -3.20 & -2.51 & -1.51 & -2.63 \\
Intermol energy & -5.29 & -4.6 & -3.6 & -4.72 \\
Vdw & -5.23 & -4.37 & -3.59 & -4.62 \\
Electrostatic energy & -0.07 & -0.22 & 0.00 & -0.1 \\
Ligand efficiency & -0.13 & -0.1 & -0.06 & -0.11 \\
Inhibit constant & 4.48 & 14.52 & 78.53 & 11.71 \\
Total internal & -1.54 & -1.54 & -0.75 & -0.83 \\
Torsional energy & 2.09 & 2.09 & 2.09 & 2.09 \\
Unbound energy & -1.54 & -1.54 & -0.75 & -0.83 \\
H-bond & 2 & 1 & 0 & 0 \\
\hline
\end{tabular}

The Blends module in Materials Studio software 8.0 is widely used to calculate the intermolecular compatibility, and it can also be used to calculate the interaction parameters for performing DPD mesoscopic simulation. In the Blends module, the mixing energy between the same molecules was set to $0 \mathrm{kcal} / \mathrm{mol}$. As shown in Table 4 , after calculation, the mixing energy between DOX and BIN was $1.533 \mathrm{kcal} / \mathrm{mol}$, which is much lower than the mixing energy 


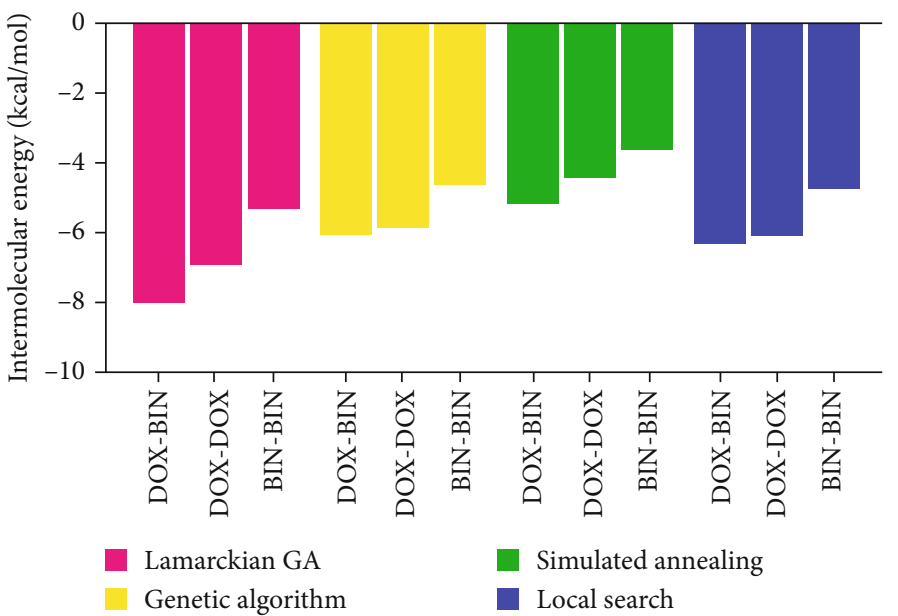

FIgUre 1: The intermolecular energy between DOX and BIN, DOX and DOX, and BIN and BIN.

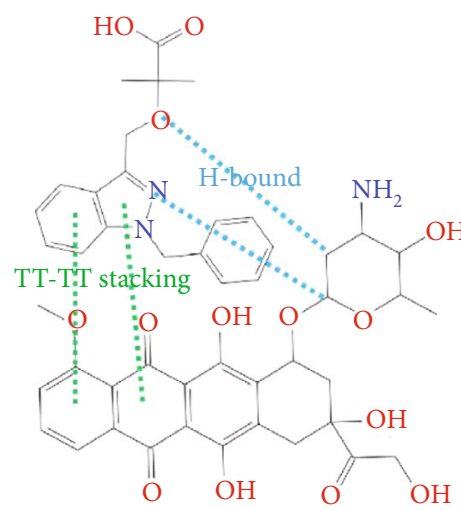

(a)

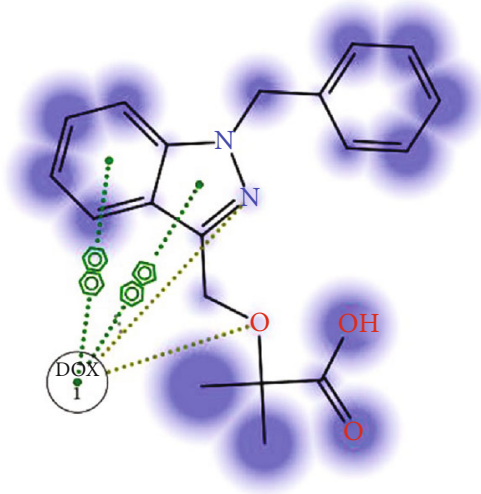

(b)

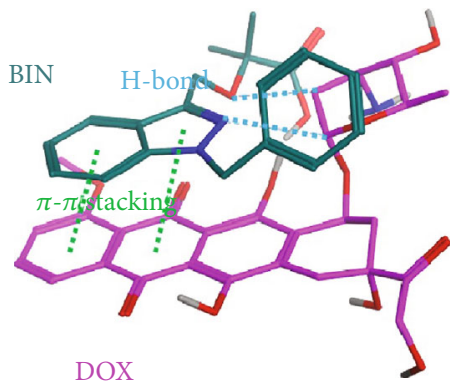

(c)

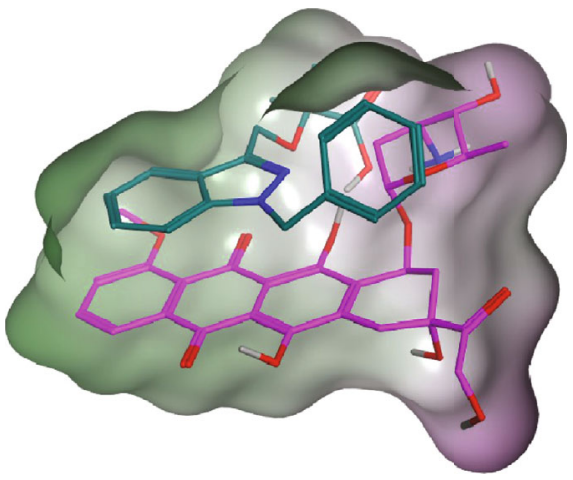

Hydrophilic

Neutral

Lipophilic

(d)

FIGURE 2: Intermolecular forces between DOX and BIN. (a) Schematic diagram between DOX and BIN; intermolecular forces between DOX and BIN were $\pi-\pi$ stacking and H-bond. (b) $2 \mathrm{D}$ conformation of BIN docked into DOX. (c) 3D conformation of complex of DOX/BIN. (d) The hydrophilic and lipophilic surface of complex of DOX/BIN.

of $52.426 \mathrm{kcal} / \mathrm{mol}$ between DOX and water and $10.768 \mathrm{kcal} / \mathrm{mol}$ between BIN and water. The compatibility between DOX and BIN is therefore much better than that between DOX and water, as well as that between BIN and water. In a Blends system of DOX, BIN, and water, DOX and BIN would combine with each other to form a complex because of their common hydrophobic properties. This is a very important basis for the formation of DOX/BIN NPs. 
TABle 4: The Flory-Huggins interaction parameters calculated by Blends module (unit: kcal/mol).

\begin{tabular}{|c|c|c|c|c|c|c|}
\hline Energies & DOX-BIN & DOX-DOX & DOX-water & BIN-BIN & BIN-water & Water-water \\
\hline$\chi^{2}(298 \mathrm{~K})$ & 1.533 & 0 & 52.426 & 0 & 10.768 & 0 \\
\hline$E_{\text {mix }}(298 \mathrm{~K})$ & 0.908 & 0 & 31.046 & 0 & 6.377 & 0 \\
\hline$E_{\mathrm{bb}}$ avg $(298 \mathrm{~K})$ & -14.95 & -14.95 & -14.95 & -7 & -7 & -1.655 \\
\hline$E_{\mathrm{bs}}$ avg $(298 \mathrm{~K})$ & -10.73 & -14.95 & -2.076 & -7 & -2.438 & -1.655 \\
\hline$E_{\mathrm{ss}}$ avg $(298 \mathrm{~K})$ & -7 & -14.95 & -1.655 & -7 & -1.655 & -1.655 \\
\hline$E_{\mathrm{bb}} \min$ & -15.854 & -15.854 & -15.854 & -9.036 & -9.036 & -2.638 \\
\hline$E_{\mathrm{bs}} \min$ & -12.06 & -15.854 & -4.885 & -9.036 & -4.762 & -2.638 \\
\hline$E_{\mathrm{ss}} \min$ & -9.036 & -15.854 & -2.638 & -9.036 & -2.638 & -2.638 \\
\hline$E_{\mathrm{bb}}$ avg & -2.615 & -2.615 & -2.615 & -1.682 & -1.682 & -0.077 \\
\hline$E_{\mathrm{bs}}$ avg & -2.106 & -2.615 & -0.537 & -1.682 & -0.435 & -0.077 \\
\hline$E_{\mathrm{ss}}$ avg & -1.682 & -2.615 & -0.077 & -1.682 & -0.077 & -0.077 \\
\hline$E_{\mathrm{bb}} \max$ & 2.336 & 2.336 & 2.336 & 5.269 & 5.269 & 3.388 \\
\hline$E_{\mathrm{bs}} \max$ & 3.638 & 2.336 & 4.966 & 5.269 & 5.228 & 3.388 \\
\hline$E_{\mathrm{ss}} \max$ & 5.269 & 2.336 & 3.388 & 5.269 & 3.388 & 3.388 \\
\hline$Z_{\mathrm{bb}}$ & 5.577 & 5.577 & 5.577 & 5.531 & 5.531 & 5.509 \\
\hline$Z_{\mathrm{bs}}$ & 6.088 & 5.577 & 12.171 & 5.531 & 11.748 & 5.509 \\
\hline$Z_{\mathrm{sb}}$ & 5.122 & 5.577 & 2.473 & 5.531 & 2.644 & 5.509 \\
\hline$Z_{\mathrm{ss}}$ & 5.531 & 5.577 & 5.509 & 5.531 & 5.509 & 5.509 \\
\hline
\end{tabular}

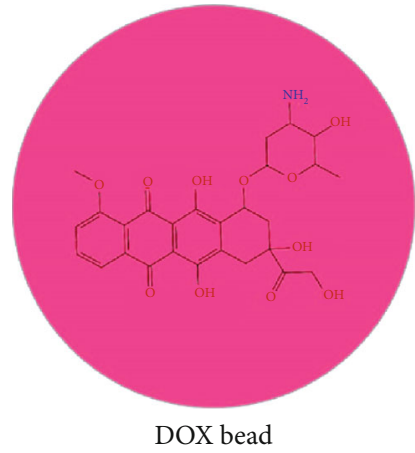

(a)

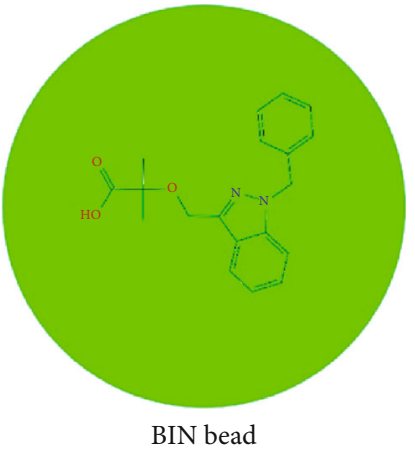

(b)

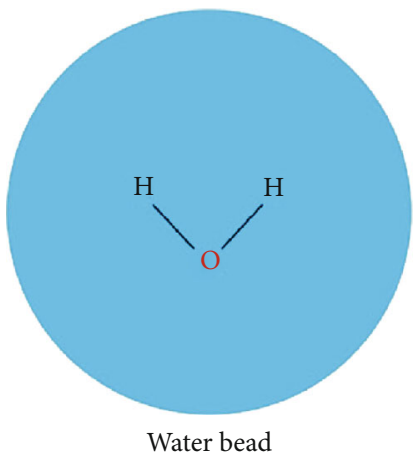

(c)

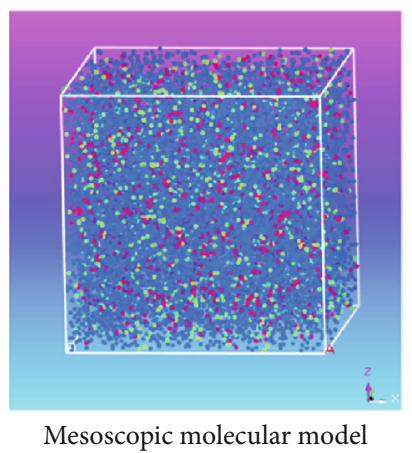

(d)

FIGURe 3: Mesoscopic molecular model: (a) DOX bead; (b) BIN bead; (c) water bead; (d) mesoscopic molecular model of beads of DOX, $\mathrm{BIN}$, and water $(1: 1: 8)$.

3.2. The Simulation of the Assembly of DOX and BIN in Water. Based on the calculated mixing energy, the FloryHuggins parameters $\left(\chi^{2}\right)$ between molecular pairs were obtained simultaneously. Then, the DOX, BIN, and water molecules were coarse-grained to the corresponding beads (Figure 3).

As shown in Table 5, according to the formula $\alpha_{\mathrm{ij}}(\rho=3)$ $=25+3.50 \cdot \chi^{2}$, the DPD repulsion parameter $\alpha_{\mathrm{ij}}$ was achieved and applied to build a DPD force field. Based on this DPD force field, a mesoscopic molecular model was constructed with beads of DOX, BIN, and water at the DOX/BIN/water ratio of $1: 1: 8$, and its conformation was further optimized. In the initial mesoscopic model, DOX, BIN, and water beads were randomly distributed in the simulated lattice (Figure 3).
TABLE 5: The interaction parameters of DPD simulation.

\begin{tabular}{lcc}
\hline Energies & $\chi^{2}(298 \mathrm{~K})$ & $\alpha_{\mathrm{ij}}$ \\
\hline DOX-BIN & 1.533 & 30.364 \\
DOX-water & 52.426 & 208.492 \\
BIN-water & 10.768 & 62.69 \\
\hline
\end{tabular}

Then, the DPD mesoscopic simulation [24] was performed using the DPD module in Mesocite for $50 \mathrm{~ns}$ to display the assembly process of DOX/BIN NPs. As shown in Figure 4, the DOX beads and BIN beads gradually changed from the randomly distributed state to small aggregates during 0-200 ps. These small aggregates first formed a core of DOX, following which BIN was gradually distributed to its 


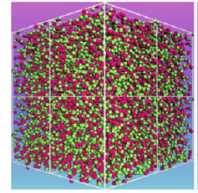

0 ps

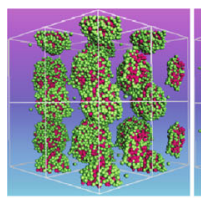

500 ps

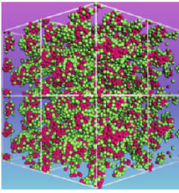

$30 \mathrm{ps}$

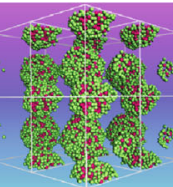

1000 ps

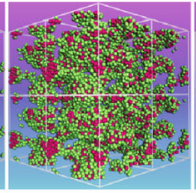

50 ps

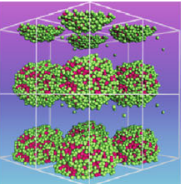

2000 ps

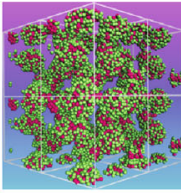

100 ps

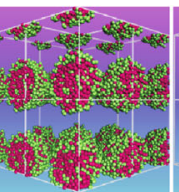

10000 ps

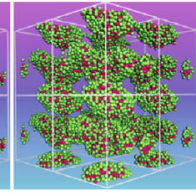

150 ps

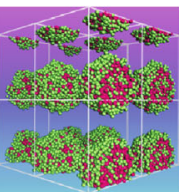

20000 ps
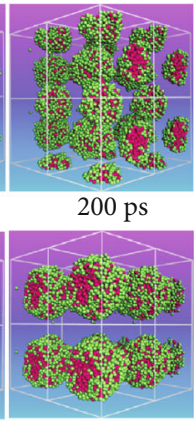

50000 ps

(a)
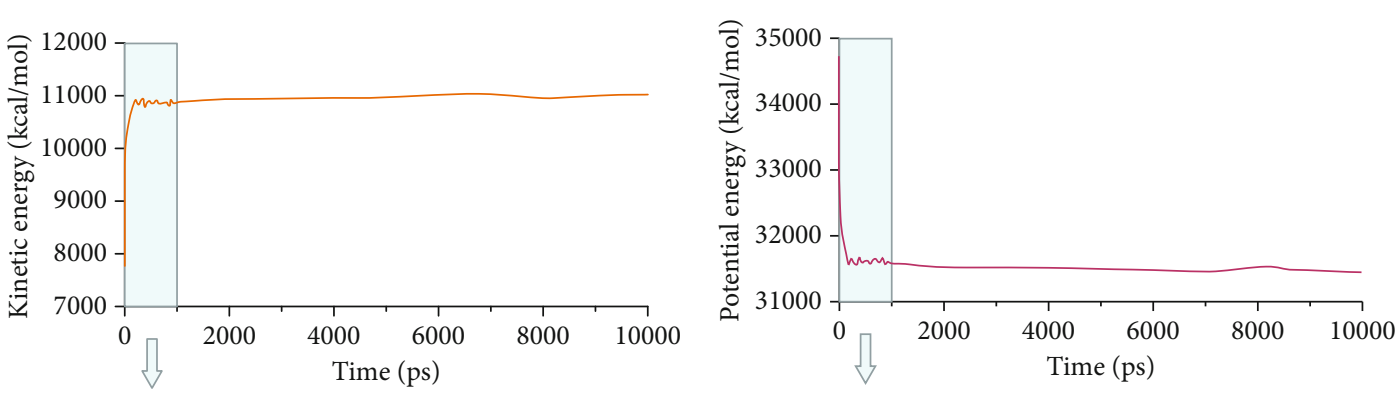

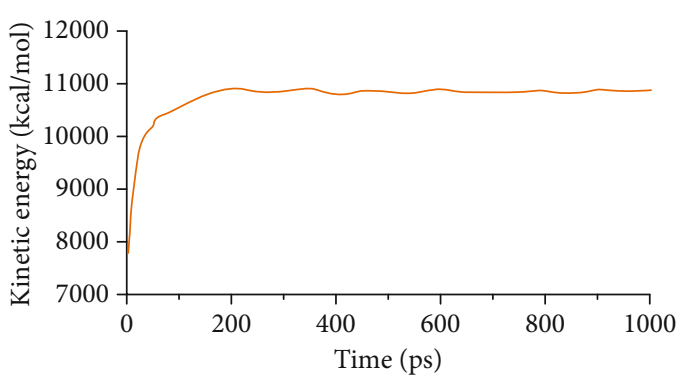

(b)

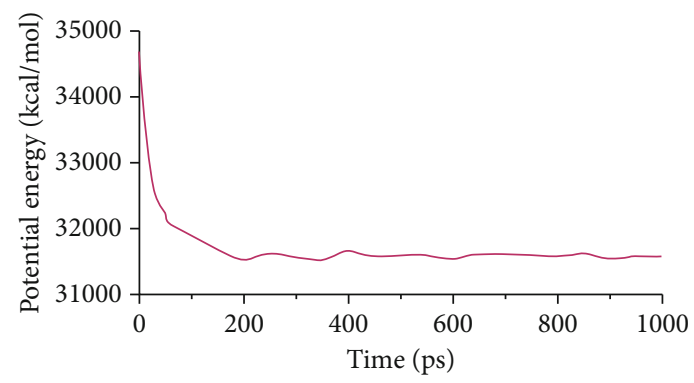

(c)
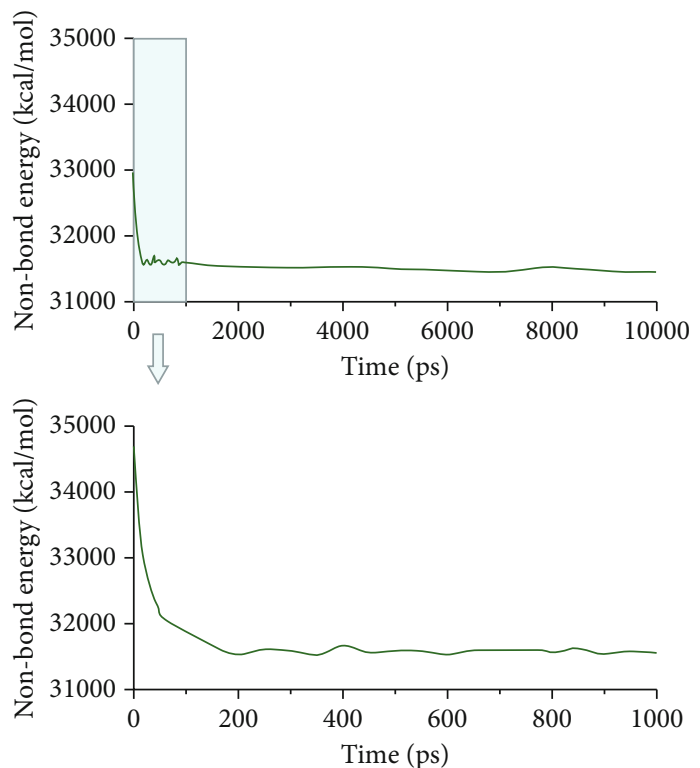

(d)

FIGURE 4: DPD mesoscopic simulation. (a) The kinect mesostructure of complex of DOX/BIN during DPD simulation from 0 to 50000 ps (red is DOX, green is BIN). (b) Kinetic energy change from 0 to 10000 ps; the figure below shows the enlargement of $0-1000$ ps. (c) Potential energy change from 0 to $10000 \mathrm{ps}$; the figure below shows the enlargement of $0-1000 \mathrm{ps}$. (d) Nonbond energy change from 0 to 10000 ps; the figure below shows the enlargement of $0-1000$ ps. 


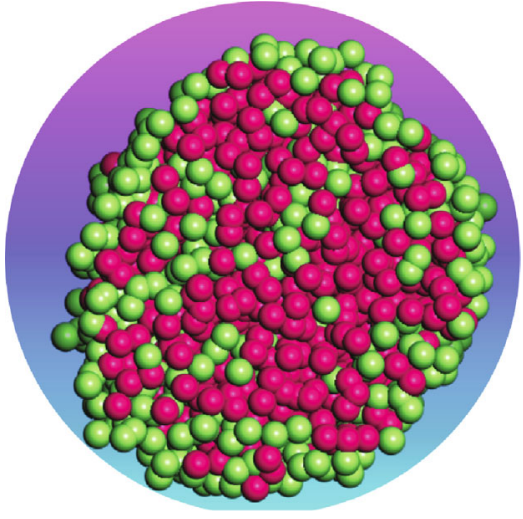

(a)

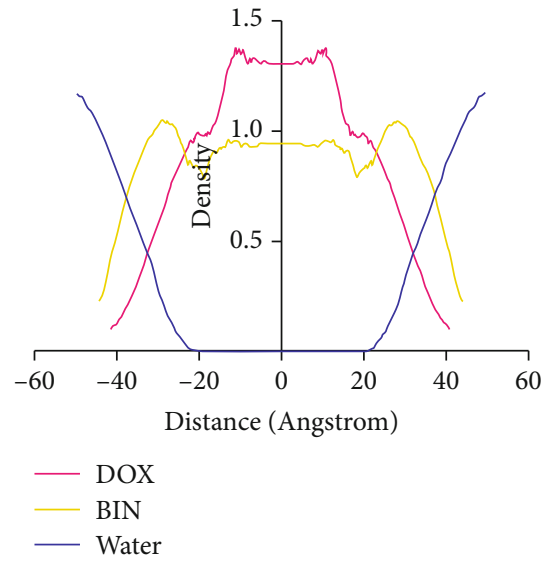

(b)

FIGURE 5: The internal structure of DOX/BIN NPs: (a) cross-section of DOX/BIN NPs; (b) molecular distance between the core and DOX, $\mathrm{BIN}$, and water.

surface. At this time, the kinetic energy increased sharply, while the theoretical energy and the nonbond energy decreased (Figures $4(\mathrm{~b})-4(\mathrm{~d})$ ). Then, the aggregates gradually assembled into spheres during $200-1000 \mathrm{ps}$, and all the energy fluctuated at its baseline. Finally, stable spherical aggregates were formed, and this structure was maintained from 1000 to $50000 \mathrm{ps}$. All the energy remained stable from 1000 to 10000 ps.

Because BIN is more hydrophilic than DOX, the crosssection of the stable spherical aggregates revealed that the DOX beads were mainly packed in the core, while the outer layer was primarily distributed with BIN beads (Figure 5). This was further confirmed by the calculation of the molecular distance.

The above DPD simulation results strongly indicate that DOX and BIN could assemble to nanoaggregates in water, and BIN molecules are mainly distributed on the surface of nanoparticles, while DOX is distributed in the core of nanoparticles.

In theory, the chemical groups of different drug molecules can be combined through various intermolecular forces, such as $\pi-\pi$ stacking, hydrogen bond, ionization, and van der Waals force. However, it is difficult to determine whether the combination is stable and whether nanoparticles are formed. Multiscale computer simulation-assisted designs have great advantages in solving the above problems [24]. Through molecular docking simulation, we can observe and compare the different intermolecular forces to verify whether the two different drug molecules tend to combine [25]. On this basis, the molecules are coarse-grained to beads through DPD mesoscopic simulation. And then, the shape and internal structure of nanoparticles can be directly observed on the computer, which provides a strong support for the preparation of nanoparticles. However, due to the limitation of computer resources, our bead setting in the DPD simulation is not fine enough to observe the distribution of intermolecular forces between DOX and BIN molecules in nanoparticles. On the basis of sufficient computer power, through the DPD simulation method based on beads representing chemical groups, the distribution of molecules in nanoparticles will be more finely simulated. By comparing with the results of all atom simulation results, it is expected to count the distribution of intermolecular forces in nanoparticles and give a more complete diagram of the internal assembly mechanism of nanoparticles [26, 27].

3.3. Characterization of DOX/BIN NPs. After mesoscopic simulation, DOX/BIN NPs were prepared by the dialysis method with a feed ratio (DOX : BIN =1:1). As shown in Figure 6, the DOX/BIN NPs presented a turbid orange solution with a granular sensation. The particle size of DOX/BIN NPs was $317.3 \pm 10.3 \mathrm{~nm}$, and the zeta-potential was 31.0 $\pm 5.9 \mathrm{mV}$. Subsequently, the TEM images of DOX/BIN NPs were scanned and presented a spherical aggregate structure. The drug loading contents of DOX and BIN were $42.06 \%$ and $57.94 \%$, respectively.

The results of molecular docking suggested that there is mainly $\pi-\pi$ stacking and hydrogen bonding between DOX and BIN. Therefore, we further characterized the intermolecular forces in DOX/BIN NPs using the UV and IR absorption spectra. The ultraviolet spectrum showed that the absorption peak of DOX was at $482 \mathrm{~nm}$, whereas the absorption peak of DOX/BIN NPs was at $516 \mathrm{~nm}$ (Figure 6(e)). This red-shift of the absorption peak indicated that the preparation of DOX/BIN NPs was successful. Fourier transform infrared spectroscopy (FT-IR) showed that the absorption bands at $3316 \mathrm{~cm}^{-1}$ of DOX, which represent the stretching vibration of the $\mathrm{O}-\mathrm{H}$ group, were significantly reduced when it was assembled with $\mathrm{BIN}$, suggesting that $\mathrm{H}$-bonding occurred. In addition, the evident decreasing of the absorbance at $739 \mathrm{~cm}^{-1}$ of BIN indicated the association of hydrophobic parts (Figure 6(f)). These results suggest that the assembly of DOX and BIN is mediated by multiple noncovalent forces, including $\pi-\pi$ stacking interactions and $\mathrm{H}$-bonds. The results of the UV and FT-IR analysis were consistent with those of the AutoDock and DPD Mesoscopic simulations and further verified that $\pi-\pi$ stacking interactions and $\mathrm{H}$-bonds were the main forces driving the assembly of DOX/BIN NPs. 


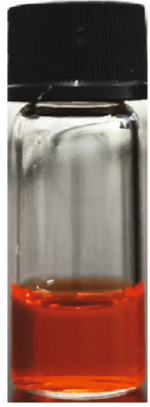

DOX

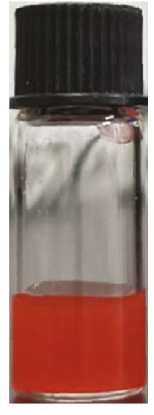

DOX/BIN NPs

(a)

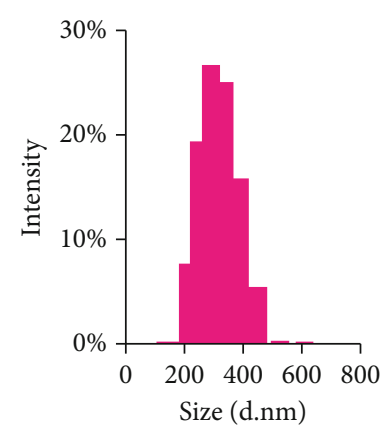

(b)

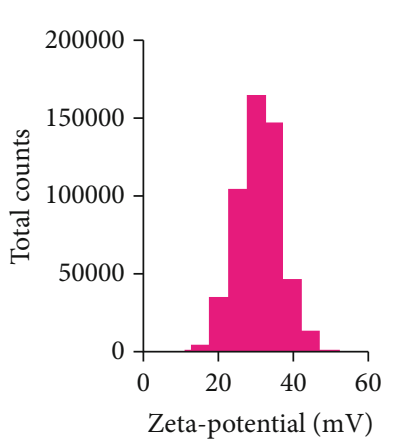

(c)

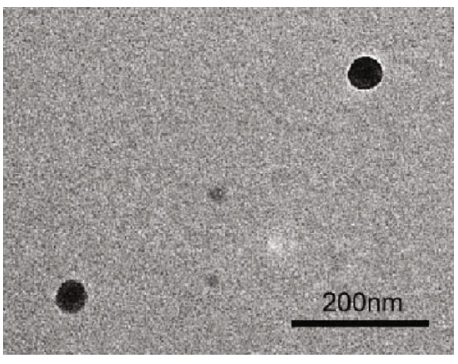

(d)

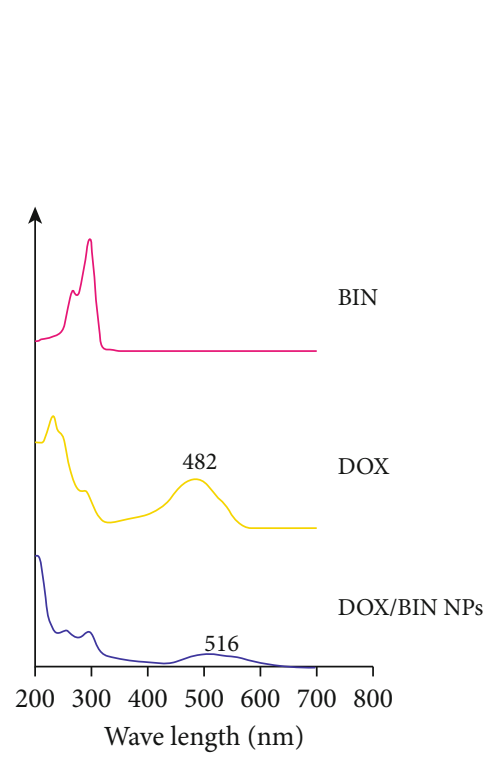

(e)

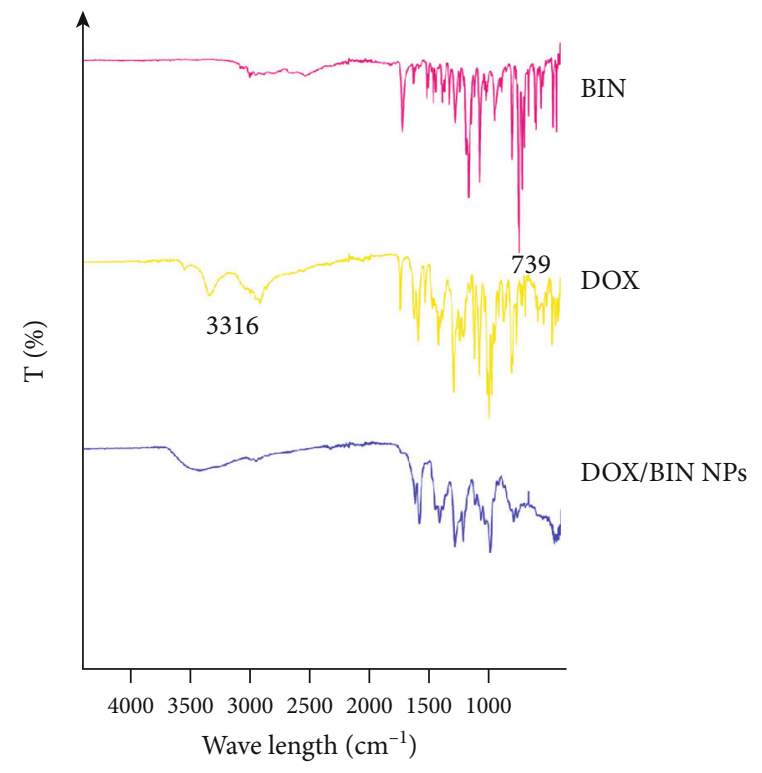

(f)

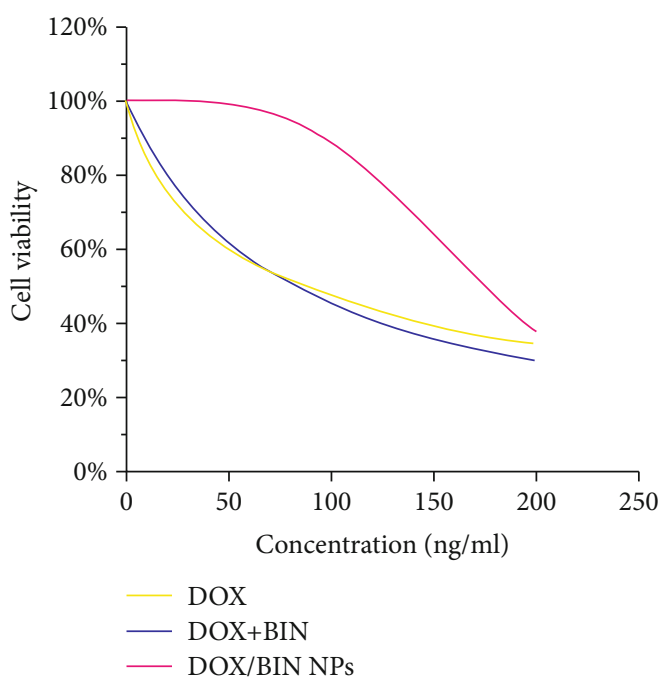

(g)

FIGURE 6: The characterization of DOX/BIN NPs. (a) DOX/BIN NPs are an orange solution with granular appearance. (b) The particle size distribution. (c) Zeta-potential distribution. (d) The TEM image of DOX/BIN NPs. (e) The ultraviolet spectrum. (f) Fourier transform infrared spectrum (FT-IR). (g) The cell viability of Y79 cells was gradually inhibited with increasing concentrations. 
3.4. Cytotoxicity of DOX/BIN NPs. To test the antitumor activity of DOX/BIN NPs, a cytotoxicity assay was performed. The viability of retinoblastoma Y79 cells was inhibited by DOX/BIN NPs, and the antitumor activity was enhanced with increasing concentrations of DOX/BIN NPs (Figure $6(\mathrm{~g})$ ). When the concentration reached $200 \mathrm{ng} / \mathrm{mL}$, the antitumor activity of DOX/BIN NPs was similar to that of DOX or DOX+BIN. The results indicated that DOX/BIN NPs might have the potential to be used in antitumor therapy.

\section{Conclusion}

In summary, a multiscale computer simulation was successfully applied to predict and design a novel doxorubicin nanomedicine with bindarit as a carrier. In this simulation, the strong intermolecular interactions $(\pi-\pi$ stacking and H-bonding) between DOX and BIN were calculated as a basis for supporting the formation of a stable DOX/BIN complex. Additionally, the hydrophilicity analysis of the DOX/BIN complex revealed an amphiphilic structure, which indicates the potential assembly ability of the DOX/BIN complex in water. A mesoscopic simulation based on DPD methodology further demonstrated that the DOX/BIN complex can self-assemble to spherical aggregates wherein DOX is concentrated internally and BIN on the outer surface. Under the guidance derived from this multiscale computer simulation, DOX/BIN NPs were successfully and facilely prepared using a dialysis method against water. The produced DOX/BIN NPs showed a good uniform nanosize distribution profile, high charged surface characteristics, and effective antitumor activity inherited from DOX. Furthermore, the intermolecular forces in DOX/BIN NPs driving the assembly were demonstrated to be multiple noncovalent forces, including $\pi-\pi$ stacking interactions and $\mathrm{H}$-bonds, which is consistent with the results from the computational simulation. Although additional studies are needed to further investigate the in vivo antitumor activity of the DOX/BIN NPs, these findings suggest that this multiscale computer simulation approach is an effective design strategy to guide the development of novel nanomedicines using a drug with synergistic antitumor activity as a carrier. This may result in high safety and low production costs and may open an alternative way to fabricate novel nanomedicines in many fields of research.

\section{Data Availability}

The data used to support the findings of this study are available from the corresponding author upon request.

\section{Conflicts of Interest}

The authors declare that there is no conflict of interest regarding the publication of this article.

\section{Acknowledgments}

This study was supported by the Natural Science Foundation Project of Chongqing, Chongqing Science and Technology Commission (Grant Nos. cstc2019jcyj-msxmX0603), Scientific Startup Fund of CQUT (Grant No. 2019ZD88), and Chongqing Science and Technology Program (Grant Nos. 30119-2961). The authors thank the Analytical \& Testing Center of Sichuan University for the computing facilities.

\section{References}

[1] S. Parveen and S. K. Sahoo, "Evaluation of cytotoxicity and mechanism of apoptosis of doxorubicin using folatedecorated chitosan nanoparticles for targeted delivery to retinoblastoma," Cancer Nanotechnology, vol. 1, no. 1-6, pp. 4762, 2010.

[2] C. Esposito, A. Crema, A. Ponzetto, G. Murtas, and G. Carloni, "Multifunctional anti-cancer nano-platforms are moving to clinical trials," Current Drug Metabolism, vol. 14, no. 5, pp. 583-604, 2013.

[3] C. R. Safinya and K. K. Ewert, "Liposomes derived from molecular vases,” Nature, vol. 489, no. 7416, pp. 372-374, 2012.

[4] V. P. Torchilin, "Recent advances with liposomes as pharmaceutical carriers," Nature Reviews Drug Discovery, vol. 4, no. 2, pp. 145-160, 2005.

[5] F. Greco and M. J. Vicent, "Combination therapy: opportunities and challenges for polymer-drug conjugates as anticancer nanomedicines," Advanced Drug Delivery Reviews, vol. 61, no. 13, pp. 1203-1213, 2009.

[6] H. Cabral and K. Kataoka, "Progress of drug-loaded polymeric micelles into clinical studies," Journal of Controlled Release, vol. 190, pp. 465-476, 2014.

[7] J. Della Rocca, D. Liu, and W. Lin, "Are high drug loading nanoparticles the next step forward for chemotherapy?," Nanomedicine, vol. 7, no. 3, pp. 303-305, 2012.

[8] C. Zhang, L. Long, Y. Xiong et al., "Facile engineering of indomethacin-induced paclitaxel nanocrystal aggregates as carrier-free nanomedicine with improved synergetic antitumor activity," ACS Applied Materials \& Interfaces, vol. 11, no. 10, pp. 9872-9883, 2019.

[9] W. Li, Y. Yang, C. Wang et al., "Carrier-free, functionalized drug nanoparticles for targeted drug delivery," Chemical Communications, vol. 48, no. 65, pp. 8120-8122, 2012.

[10] F. Yang, S. S. Teves, C. J. Kemp, and S. Henikoff, "Doxorubicin, DNA torsion, and chromatin dynamics," Biochimica et Biophysica Acta (BBA) - Reviews on Cancer, vol. 1845, no. 1, pp. 84-89, 2014.

[11] K. Shimizu, H. Kawashima, A. Kawai, M. Yoshida, and Y. Nishida, "Effectiveness of doxorubicin-based and liposomal doxorubicin chemotherapies for patients with extraabdominal desmoid-type fibromatosis: a systematic review," Japanese Journal of Clinical Oncology, vol. 50, no. 11, pp. 1274-1281, 2020.

[12] K. B. Knudsen, H. Northeved, P. Kumar EK et al., "In vivo toxicity of cationic micelles and liposomes," Nanomedicine: Nanotechnology, Biology and Medicine, vol. 11, no. 2, pp. 467-477, 2015.

[13] L. Yin, C. Peng, Y. Tang et al., "Biomimetic oral targeted delivery of bindarit for immunotherapy of atherosclerosis," Biomaterials Science, vol. 8, no. 13, pp. 3640-3648, 2020. 
[14] S. Oddi, L. Scipioni, A. Totaro et al., "The anti-inflammatory agent bindarit acts as a modulator of fatty acid- binding protein 4 in human monocytic cells," Scientific Reports, vol. 9, no. 1, p. 15155, 2019.

[15] C. Xu, L. Yin, Z. Teng et al., "Prevention of obesity related diseases through laminarin-induced targeted delivery of bindarit,” Theranostics, vol. 10, no. 21, pp. 9544-9560, 2020.

[16] M. Zollo, V. di Dato, D. Spano et al., "Targeting monocyte chemotactic protein-1 synthesis with bindarit induces tumor regression in prostate and breast cancer animal models," Clinical \& Experimental Metastasis, vol. 29, no. 6, pp. 585-601, 2012.

[17] J. L. Steiner, J. M. Davis, J. L. McClellan, A. Guglielmotti, and E. A. Murphy, "Effects of the MCP-1 synthesis inhibitor bindarit on tumorigenesis and inflammatory markers in the C3(1)/SV40Tag mouse model of breast cancer," Cytokine, vol. 66, no. 1, pp. 60-68, 2014.

[18] M. K. Shim, J. Park, H. Y. Yoon et al., "Carrier-free nanoparticles of cathepsin B-cleavable peptide-conjugated doxorubicin prodrug for cancer targeting therapy," Journal of Controlled Release, vol. 294, pp. 376-389, 2019.

[19] T. T. Talele, S. A. Khedkar, and A. C. Rigby, "Successful applications of computer aided drug discovery: moving drugs from concept to the clinic," Current Topics in Medicinal Chemistry, vol. 10, no. 1, pp. 127-141, 2010.

[20] X. Zhou, Y. Zhao, S. Chen et al., "Self-assembly of pHresponsive microspheres for intestinal delivery of diverse lipophilic therapeutics," Biomacromolecules, vol. 17, no. 8, pp. 2540-2554, 2016.

[21] L. Che, Z. Liu, D. Wang et al., "Computer-assisted engineering of programmed drug releasing multilayer nanomedicine via indomethacin-mediated ternary complex for therapy against a multidrug resistant tumor," Acta Biomaterialia, vol. 97, pp. 461-473, 2019.

[22] N. T. Nguyen, T. H. Nguyen, T. N. H. Pham et al., "AutoDock Vina adopts more accurate binding poses but AutoDock4 forms better binding affinity," Journal of Chemical Information and Modeling, vol. 60, no. 1, pp. 204-211, 2020.

[23] G. Bitencourt-Ferreira, V. O. Pintro, and W. F. de Azevedo, "Docking with AutoDock4," in Docking Screens for Drug Discovery, W. F. Azevedo Jr., Ed., pp. 125-148, Springer New York, New York, NY, 2019.

[24] Y. H. Feng, X. P. Zhang, Z. Q. Zhao, and X. D. Guo, “Dissipative particle dynamics aided design of drug delivery systems: a review," Molecular Pharmaceutics, vol. 17, no. 6, pp. 17781799, 2020.

[25] T. Kaur, A. Madgulkar, M. Bhalekar, and K. Asgaonkar, "Molecular docking in formulation and development," Current Drug Discovery Technologies, vol. 16, no. 1, pp. 30-39, 2019.

[26] M. Tekpinar and W. Zheng, "Coarse-grained and all-atom modeling of structural states and transitions in hemoglobin," Proteins: Structure, Function, and Bioinformatics, vol. 81, no. 2, pp. 240-252, 2013.

[27] K. Y. Sanbonmatsu and C. S. Tung, "High performance computing in biology: multimillion atom simulations of nanoscale systems," Journal of Structural Biology, vol. 157, no. 3, pp. 470480, 2007. 